\title{
La integridad académica entre el alumnado universitario español*
}

\author{
Academic integrity among Spanish university students \\ Integridade acadêmica entre os estudantes universitários espanhóis \\ Rubén Comas ${ }^{1}$, Jaume Sureda ${ }^{2}$, Antonio Casero ${ }^{3}$, Mercè Morey $^{4}$ \\ ${ }^{1}$ (Universidad de las Islas Baleares) rubencomas@uib.es. Fono: 0034971172378 \\ 2(Universidad de las Islas Baleares) jaume.sureda@uib.es. Fono: 0034971172378 \\ ${ }^{3}$ (Universidad de las Islas Baleares) vdceacm0@uib.es. Fono: 0034971172378 \\ ${ }^{4}$ Mercè Morey (Universidad de las Islas Baleares) merce.morey@uib.es. Fono: 0034971172378
}

\begin{abstract}
RESUMEN
La investigación que se expone a través de este artículo tuvo como principal finalidad realizar un estudio exhaustivo acerca de la deshonestidad académica entre el alumnado universitario español. Para dar respuesta a las cuestiones planteadas se diseñó una investigación de ámbito nacional a través de una encuesta transversal dirigida a la población objeto de estudio. La muestra, basada en el procedimiento de muestreo no probabilístico, estuvo compuesta por un total de 560 alumnos. De los resultados de la investigación se puede destacar que no existe un uso substancial de las TIC en las prácticas deshonestas del alumnado a la hora de realizar exámenes escritos; mientras que, en relación a las prácticas deshonestas ligadas a la elaboración de trabajos, se constata que Internet se ha convertido en la fuente principal del alumnado a la hora de plagiar trabajos académicos.
\end{abstract}

Palabras clave: deshonestidad académica, plagio, actitudes de los estudiantes, integridad académica, ciberplagio.

\begin{abstract}
One of the main goals of this research is to carry out a comprehensive study on the academic dishonesty practices among Spanish university students. To answer the research questions we designed a national investigation based on a cross-sectional survey aimed at the targeted population. The sample consisted of a total of 560 students and the procedure was a non-probability sampling. The research findings do not show substantial use of ICT in dishonest practices when replying to written exams. Ion the other hand, Internet has become the main source of students for the plagiarism of academic essays and papers.
\end{abstract}

Key words: academic dishonesty, plagiarism, student attitudes, academic integrity, cyber-plagiarism.

\section{RESUMO}

A pesquisa descrita neste artigo teve como objetivo principal fazer um estudo exaustivo sobre a desonestidade acadêmica entre estudantes universitários espanhóis. Para responder às perguntas fizemos uma pesquisa nacional projetada através de um estudo transversal. A amostra, com base em procedimentos de amostragem não probabilística, foi respondida por um total de 560 alunos. A partir dos resultados da investigação pode-se notar que nao há uso sustancial das TIC nas práticas desonestas durante a condução das provas escritas, enquanto em relação a práticas desonestas ligadas ao desenvolvimento do trabalho, é claro que a Internet se tornou a principal fonte de estudantes para plagiar os trabalhos acadêmicos.

Palavras-chave: desonestidade acadêmica; plágio; atitudes dos alunos; integridade acadêmica; cyber-plágio.

* El presente artículo ha sido elaborado en el marco del proyecto de I+D financiado por el MEC “El ciberplagio entre los estudiantes universitarios" con Referencia SEJ2006-10413. Los autores agradecen al MCyT el apoyo concedido. De la misma manera hacen extensiva su gratitud para con el portal Universia por la colaboración dispensada. 


\section{INTRODUCCION}

Como sostiene Kibler (1993), una de las principales dificultades a la hora de encarar el tema de la deshonestidad académica ${ }^{1}$ es la falta de una definición unívoca y clara del concepto ya que se trata de un constructo basado en principios ético-morales y, por consiguiente, asociado a una época, una cultura y una sociedad determinadas. A fin de paliar, en la medida de lo posible, esta dificultad, a modo de exordio, hemos optado por sistematizar aquellos comportamientos y prácticas desarrolladas por el alumnado ${ }^{2}$ catalogadas como deshonestas en las que se han centrado diversos autores (Brimble \& Stevenson-Clarke, 2005; Jeffers, 2002; Lambert, Hogan \& Barton, 2003; McCabe \& Trevino, 1993; Moeck, 2002) e instituciones académicas que han abordado el tema. Así, hemos comprobado que las principales acciones deshonestas consideradas en la literatura existente corresponden, básicamente, a tres grandes ámbitos: a) el de los exámenes; b) el de la elaboración y presentación de trabajos; y, finalmente, c) el referido a las interrelaciones con los compañeros. En la tabla 1 hemos sistematizado y clasificado las acciones definitorias de la deshonestidad académica para cada una de las tres áreas.

Los estudios existentes acerca de la deshonestidad académica entre universitarios se centran, principalmente, en el análisis de cuatro grandes ámbitos de investigación, siendo: análisis de la prevalencia y profundidad del fenómeno -campo en que se enmarca el presente artículo-; análisis de las causas; análisis centrados en el perfil de los infractores de las normas académicas y, finalmente, aquellos orientados a la detección y reducción del fraude académico ${ }^{3}$.

La literatura sobre el análisis de la prevalencia de acciones contra la integridad académica se desarrolla a partir de trabajos que, eminentemente, intentan medir cuán extendida está la deshonestidad académica entre la población universitaria. En este tipo de aproximaciones se procuran calcular aspectos relacionados con prácticas que atentan contra la integridad académica (por ejemplo: copiar en exámenes, plagiar trabajos usando Internet, plagio de trabajos ya presentados, compraventa de trabajos, etc.) y dependiendo del autor se da mayor énfasis a un aspecto que a otro. Hay trabajos, por ejemplo, interesados exclusivamente en análisis relativos al comportamiento deshonesto del alumnado en el transcurso de pruebas escritas (Bowers, 1964; Davis, Pierce, Yandell \& Arnow, 1995; Diekhoff, LaBeff, Clark, Williams, Francis \& Haynes, 1996; Genereux \& McLeod, 1995;

1 Se emplea el concepto "Deshonestidad Académica" siguiendo así la nomenclatura instaurada y empleada en los países anglosajones a través del término "Academic Dishonesty" que está íntimamente ligado a otro concepto fundamental a la hora de presentar y dar sentido a este trabajo: "Integridad Académica", entendida como la adopción y adecuación de los valores fundamentales que deben regir toda actividad académica, siendo éstos: honestidad, confianza, responsabilidad, respeto y justicia o equidad (University of San Diego, 1997).

2 En este trabajo únicamente se analizan y describen actividades o prácticas que pueden ser definidas como incorrectas o deshonestas en entornos académicos en las que el sujeto activo (por acción, omisión o facilitación) sea el alumnado; dejando fuera del análisis o consideración las actividades que pueden ser calificadas como deshonestas o incorrectas en entornos académicos llevadas a cabo por docentes y/o demás personal de instituciones académicas. La tarea se centra, de esta manera, en uno de los cuatro ámbitos descritos por la literatura existente como áreas de deshonestidad académica: el aprendizaje; no abordando los otros tres campos posibles: la gestión, la enseñanza y la investigación (Hinman, 2002).

3 Podría hablarse de un quinto ámbito de análisis sobre el que se está comenzando a investigar: las consecuencias futuras de la comisión de prácticas académicamente deshonestas en el mundo profesional. No se ha incluido entre los cuatro grandes ámbitos descritos por el hecho de que se trata de un campo de investigación naciente y con escasa documentación. Destacan, en este campo, los trabajos de Harding (2001 y 2007). 


\section{Tabla I}

Listado de acciones académicamente deshonestas e incorrectas

\begin{tabular}{|c|c|}
\hline $\begin{array}{l}\text { Acciones y prácticas aca- } \\
\text { démicamente incorrectas } \\
\text { relativas al desarrollo de } \\
\text { exámenes }\end{array}$ & $\begin{array}{l}\text { - Copiar de otro alumno en el transcurso de un examen } \\
\text { - Copiar de "chuletas" en el transcurso de un examen } \\
\text { - Usar aparatos o medios tecnológicos (teléfono móvil, reproductores } \\
\text { de MP3, PDA, etc.) para copiar en el transcurso de un examen } \\
\text { - Dejarse copiar por otro alumno en el transcurso de un examen } \\
\text { - Permitir que otra persona le suplante durante un examen } \\
\text { - Hacer un examen suplantando a otro alumno } \\
\text { - Hacerse, de manera fraudulenta, con el contenido de un examen } \\
\text { antes de realizarlo }\end{array}$ \\
\hline $\begin{array}{l}\text { Acciones y prácticas aca- } \\
\text { démicamente incorrectas } \\
\text { relativas a la elaboración } \\
\text { y presentación de trabajos } \\
\text { académicos }\end{array}$ & $\begin{array}{l}\text { - Ciber-plagio: } \\
\text { - Copiar de páginas Web u otros recursos accesibles en la Red } \\
\text { fragmentos de textos y -sin citar- pegarlos directamente en un } \\
\text { documento - en el que hay parte de texto original- y entregarlo } \\
\text { como trabajo de una asignatura } \\
\text { - Elaborar, íntegramente, un trabajo a partir de fragmentos co- } \\
\text { piados literalmente de páginas Web y/o recursos localizados en } \\
\text { Internet } \\
\text { - Descargar un trabajo completo desde Internet y entregarlo, sin } \\
\text { cambios, como trabajo de una asignatura } \\
\text { - Plagio de fuentes impresas } \\
\text { - Copiar y no citar fragmentos de textos y documentos impresos } \\
\text { (libros, periódicos, revistas, etc.) } \\
\text { - Copiar partes de trabajos entregados en años anteriores (bien } \\
\text { sean propios o sean de otro estudiante) y entregarlos como } \\
\text { partes de un trabajo académico "nuevo" } \\
\text { - Entregar un trabajo completo realizado por otro alumno que } \\
\text { ya haya sido entregado en cursos anteriores (para la misma u } \\
\text { otra/s asignatura/s) } \\
\text { - Entregar un trabajo completo realizado por uno mismo que ya } \\
\text { haya sido entregado (para la misma u otra asignatura) } \\
\text { - Facilitar a otro alumno un trabajo de años anteriores o actual para } \\
\text { que lo entregue como un trabajo propio, original e inédito } \\
\text { - Elaborar un trabajo académico para que lo entregue otra } \\
\text { persona } \\
\text { - Compraventa de trabajos académicos } \\
\text { - Falsear la bibliografía y recursos consultados en la elaboración } \\
\text { de un trabajo académico } \\
\text { - Falsear datos y resultados en trabajos académicos } \\
\text { - Colaborar en la elaboración de un trabajo sin estar permitido }\end{array}$ \\
\hline $\begin{array}{l}\text { Conductas o prácticas } \\
\text { deshonestas hacia el resto } \\
\text { de alumnado }\end{array}$ & $\begin{array}{l}\text { - Dañar el/los trabajo/s y/o material de otros alumnos } \\
\text { - Interferir en el trabajo o examen de otro alumno y perturbar su } \\
\text { actividad }\end{array}$ \\
\hline
\end{tabular}

Fuente: Elaboración propia. 
Haines, Diekhoff, LaBeff \& Clark, 1986; Rogers, 2006; Tibbetts, 1998; Whitley, 1998); mientras que otros intentan abordar la integridad académica en su globalidad y analizan -además de la deshonestidad en el transcurso de exámenes- aspectos como el plagio, la falsificación de datos y resultados en trabajos, etc. (Ashworth, Bannister \& Thorne, 1997; Brimble et al., 2005; Joyce, 2007; Lin \& Wen, 2007; McCabe, 2001; Underwood \& Szabo, 2003).

Existe escasísima bibliografía sobre la integridad académica del alumnado universitario español. Es éste un tema que, hasta el momento, ha sido escasamente tratado y del que sólo existen tres referencias destacables. La primera es la del trabajo de Rey-Abella y otros (2006), centrado en el análisis de diversas formas de deshonestidad académica entre el alumnado de la Escuela Universitaria de Enfermería, Fisioterapia y Nutrición Blanquerna. El segundo es la de un reciente trabajo auspiciado por la empresa francesa de desarrollo de software antiplagio Six Degrés (Agnes, 2008) basado en la administración de un cuestionario a 299 universitarios y 53 docentes de las universidades de Barcelona y Zaragoza. El tercer trabajo, y el más completo de los llevados a cabo en España en este campo hasta la fecha, se realizó estudiando una muestra representativa $(\mathrm{n}=727)$ del alumnado pregraduado de todos los estudios de la Universidad de las Islas Baleares (Sureda et al., 2008). Son, también, poco numerosos los trabajos de investigación llevados a cabo en otros países hispanoamericanos; de entre los que existen conviene destacar las aportaciones de: Mejías y Ordóñez (2004) que analizaron la cuestión de la honestidad académica entre el alumnado de la universidad colombiana de los Andes; en Brasil sobresalen los trabajos de investigación de Da Costa y otros (2006) sobre las malas prácticas académicas entre alumnado de Medicina de la Universidad de Salvador de Bahía y el estudio acerca de la percepción del plagio académico por parte de alumnado de primer y quinto curso de Ingeniería de la Universidad de Río de Janeiro (Garcia-Barbastefano \& Gomes-de-Souza, 2007); finalmente, en Perú se llevó a cabo (Saldaña et al., 2010) un interesante trabajo en el que se analizó la prevalencia de la comisión de plagio académico en las tesis de medicina presentadas en una universidad pública del país.

\section{METODO}

El trabajo que a continuación presentamos indaga acerca de cuáles son las principales prácticas académicas deshonestas realizadas por el alumnado pregraduado universitario español, y con qué frecuencia éstas son llevadas a cabo. El carácter exploratorio del problema de investigación, junto con la información recogida en el marco de referencia, han aconsejado el diseño de una investigación de ámbito nacional basada en una encuesta transversal, dirigida a la población objeto de estudio -universitarios pregraduados españoles- a través del portal universitario de Internet Universia (http:// www.universia.es).

Siguiendo la clasificación establecida por Comas (2009), podemos encasillar la investigación que aquí presentamos como: desarrollada en un solo país; en varias universidades (multicampus); basada en datos aportados por el alumnado de pregrado, de diversas titulaciones y que cursan estudios en modalidad presencial. 


\section{MUESTRA}

La muestra del estudio se compone de un total de 560 alumnos, adscritos todos ellos al portal Universia, presentando una media de antigüedad en la universidad española de 4,95 años y una desviación estándar de 2,69. En cuanto a la distribución de la muestra por género, comentar que: el 59,3\% de los encuestados fueron mujeres y el 40,7\% hombres. Por cursos, la muestra se desglosa en: $15,7 \%$ alumnado de primer curso; 36,4\% alumnado de segundo curso; 24,9\% alumnado de tercer curso; 17,4\% alumnado de cuarto curso; y $5,6 \%$ alumnado de quinto curso.

El procedimiento de muestreo es no probabilístico, obteniendo una muestra de voluntarios que, aun limitando las posibilidades de generalización, permite cubrir la aproximación exploratoria fijada anteriormente. El error muestral calculado para el conjunto de la muestra es de $\pm 4,11 \%{ }^{4}$, para un nivel de confianza del $95 \%$, y bajo el supuesto de muestreo aleatorio simple, y la situación más desfavorable en la que $\mathrm{p}=\mathrm{q}$.

\section{INSTRUMENTO DE RECOGIDA DE DATOS}

La recogida de los datos se realizó por medio de un cuestionario de elaboración propia, en formato digital, y enviado por correo electrónico a todos los usuarios españoles registrados en el portal Universia. En el contenido del cuestionario se presentaban los 17 escenarios (situaciones o prácticas académicas deshonestas) expuestos en la tabla 2, duplicando su presentación a fin de recoger las dos respuestas de interés para la investigación:

Frecuencia declarada: el alumno debe indicar con qué frecuencia realiza cada una de las prácticas propuestas en los escenarios. Las respuestas pueden ser: nunca, entre 1 y dos veces, entre 3 y 4 veces, entre 5 y 10 veces y en más de 10 ocasiones.

Frecuencia atribuida: el alumno debe indicar con qué frecuencia cree que sus compañeros realizan las prácticas propuestas en los escenarios. Las respuestas pueden ser: nunca, esporádicamente, frecuentemente y muy frecuentemente.

Además de las dos tablas, con el inventario de escenarios en cada una de ellas, también se solicitaba información relativa a las fuentes de plagio académico mediante la siguiente redacción: "Desde tu experiencia y conocimiento, los estudiantes universitarios a la hora de copiar trabajos (o fragmentos de trabajos) usan con mayor frecuencia”. Siendo las siguientes las cuatro opciones de respuesta: recursos que encuentran en Internet, recursos impresos (libros, revistas, periódicos, etc.), trabajos ya entregados, y otro (por favor, especifica...).

\section{PROCESO DE DATOS}

Se realizó el vaciado de toda la información contenida en los cuestionarios cumplimentados mediante el programa estadístico SPSS versión 15; se describieron las variables, etiquetas y valores, se obtuvieron y organizaron los datos estadísticos, se realizaron los

$4 \quad$ Universo: 37.490 usuarios registrados en Universia. 


\section{Tabla 2}

Escenarios propuestos según dimensiones

\section{Prácticas académicas deshonestas relativas al desarrollo de exámenes}

- Copiar de un/a compañero/a durante un examen.

- Utilizar "chuletas" en un examen.

- Utilizar nuevas tecnologías (móvil, auriculares inalámbricos,...) para obtener las respuestas en un examen.

- Dejar que otro estudiante me copie durante un examen.

- Hacer un examen en nombre de otra persona.

\section{Plagio sobre Internet (ciberplagio)}

- Copiar de páginas Web fragmentos de textos y -sin citar- pegarlos directamente en un documento -en el que hay parte de texto escrito por mí mismo/a- y entregarlo como trabajo de una asignatura.

- Componer íntegramente un trabajo a partir de fragmentos copiados literalmente de páginas Web y/o artículos localizados en Internet.

- "Descargar" un trabajo completo desde Internet y entregarlo, sin cambios, como trabajo de una asignatura.

\section{Plagio de fuentes impresas}

- Copiar fragmentos de fuentes impresas (libros, periódicos, artículos de revista, etc.) y usarlos -sin citar- para elaborar trabajos académicos propios.

\section{Plagio de trabajos académicos}

- Copiar partes de trabajos entregados en años anteriores (bien seas tú el autor/a o sea otro/a estudiante) y usarlos como partes de un trabajo académico "nuevo".

- Entregar un trabajo realizado por otro/a alumno/a que ya había sido entregado en cursos anteriores (para la misma $\mathrm{u}$ otra/s asignatura/s).

- Entregar un trabajo realizado por mí mismo/a que ya había sido entregado en cursos anteriores (para la misma u otra/s asignatura/s).

- Facilitar a un/a compañero/a un trabajo de años anteriores para que lo entregue como un trabajo nuevo e inédito.

- Hacer un trabajo para otra persona (ya sea de tu curso o de otro curso).

- Pagar a alguien para que haga un trabajo académico o comprarlo (por ejemplo a través de Internet).

\section{Falseamiento de las referencias bibliográficas}

- Insertar en el apartado bibliográfico de un trabajo referencias que realmente no se han consultado.

\section{Falseamiento de datos}

- Falsear datos en trabajos académicos (por ejemplo: falsear datos para resolver un problema en un trabajo de física, inventar datos en una investigación, presentar datos de otros trabajos inventados, etc.) 
gráficos y tablas a partir de los datos anteriores, se actualizó toda la información (frecuencias y gráficos) decidiendo el tipo de herramienta gráfica más adecuada para cada variable. Fue menester recategorizar algunas variables y tratar a otras como respuestas múltiples. Se depuraron las tablas que contenían datos erróneos y casos perdidos. Se agruparon ciertas variables y se recodificaron otras.

\section{RESULTADOS}

Los resultados de la investigación que presentamos a continuación siguen una estructura dimensional, donde el análisis de las diversas prácticas académicas deshonestas se ha organizado en torno a las siguientes dimensiones: comportamiento deshonesto en exámenes, ciberplagio, plagio de fuentes impresas, plagio de trabajos académicos, falseamiento de bibliografía. A su vez, y dentro de cada dimensión, se presenta primero la información relativa a la frecuencia con que el propio alumnado declara haber cometido el fraude académico propuesto en los distintos escenarios, y a continuación la frecuencia atribuida a los compañeros en relación a los mismos aspectos. Por último, y después de mostrar los hallazgos en torno las fuentes del plagio, presentamos el análisis de la consistencia de las prácticas con el fin de poder establecer la ordenación de las mismas a modo de ranking o clasificación.

\section{PRACTICAS ACADEMICAS DESHONESTAS RELATIVAS AL DESARROLLO DE EXAMENES}

Tal y como recoge el gráfico 1, la práctica deshonesta realizada por el alumnado universitario con mayor frecuencia es la de permitir copiar a otro estudiante del propio examen; casi tres cuartas partes del alumnado dice haberlo permitido alguna vez $(54,7 \%$ entre 1 y 4 veces y un 19,7\% 5 o más veces). Este dato es consistente en relación a que la mayor frecuencia de práctica deshonesta atribuida a los compañeros es también dejarse copiar en un examen (ver gráfico 2). Sin embargo, en este caso el porcentaje resulta ser mayor aún; casi el $90 \%$ del alumnado considera que sus compañeros alguna vez lo han permitido (33,7\% esporádicamente y el $55,1 \%$ frecuentemente o muy frecuentemente).

Copiar a un compañero durante un examen aparece como la segunda práctica académicamente deshonesta más frecuente entre el alumnado durante la realización de una prueba escrita; el 46,4\% del alumnado preguntado afirma haberlo hecho en al menos una ocasión (ver gráfico 1), y el $88,1 \%$ (ver gráfico 2) considera que esta acción es al menos una práctica esporádica entre sus compañeros. Algo similar ocurre con la utilización de chuletas o anotaciones personales durante la realización de un examen, ocupando el tercer lugar en el ranking de prácticas deshonestas tanto declaradas como atribuidas, presentando valores muy cercanos a los observados anteriormente en la acción de copiar a un compañero. En relación a estas prácticas, que ocupan las tres primeras posiciones durante la realización de un examen, llama la atención que la atribución a los compañeros como práctica frecuente o muy frecuente supera, en los tres casos, el $50 \%$.

En relación a la utilización de medios tecnológicos (teléfono móvil, MP3, radiotransmisores, PDA, etc.) para obtener las respuestas de un examen, los resultados muestran que, atendiendo a lo declarado en primera persona, se trata de una práctica con presencia marginal, tan sólo el 2,5\% del alumnado dice haber hecho uso al menos una vez de este 


\section{Gráfico 1}

Frecuencia declarada de comisión de prácticas académicas deshonestas en el transcurso de exámenes

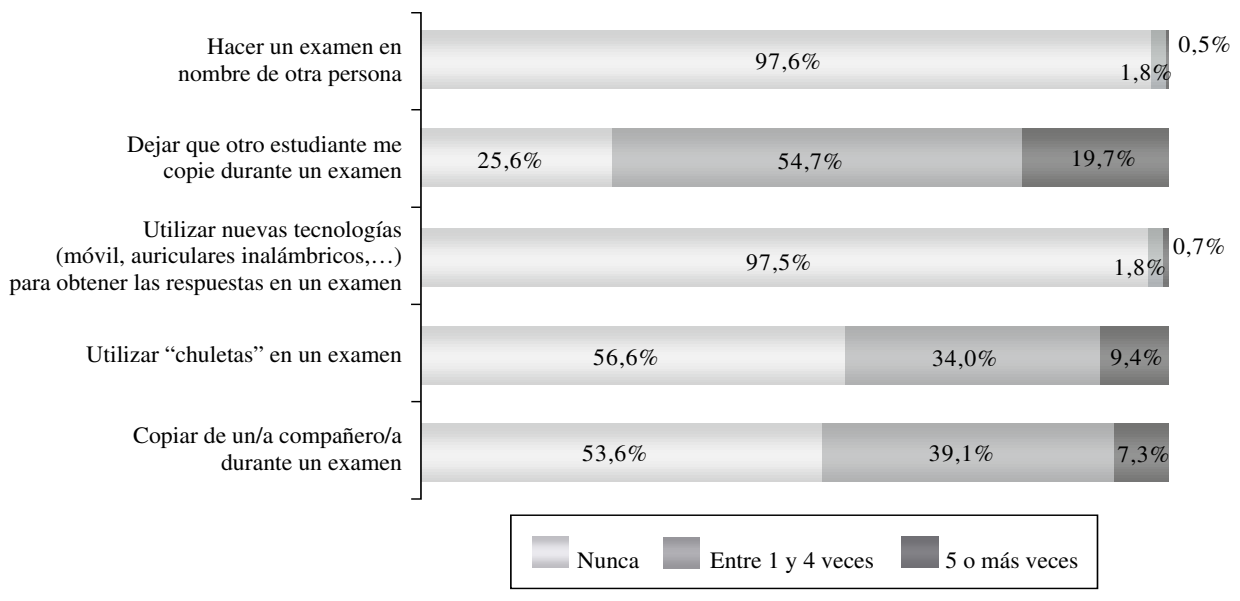

Gráfico 2

Frecuencia atribuida a los compañeros sobre la realización de las prácticas académicas deshonestas en exámenes

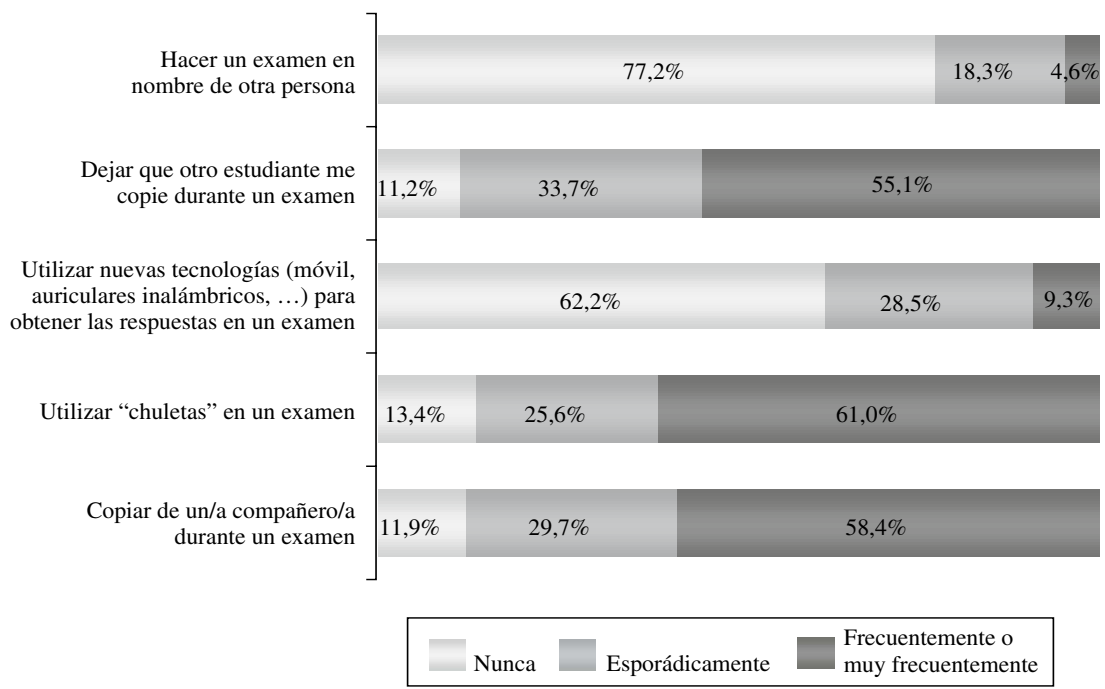

tipo de recursos. Sin embargo, el porcentaje sube hasta un 37,8\% en cuanto a la atribución de este comportamiento a los compañeros.

Una distribución de la respuesta muy parecida se observa en la práctica menos realizada de las expuestas en el cuestionario en esta dimensión: suplantar a otro en un 
examen. Si bien tan sólo un 2,4\% del alumnado dice haberlo hecho alguna vez, el 22,8\% del alumnado atribuye a sus compañeros la realización de esta práctica esporádicamente, al menos.

\section{PLAGIO USANDO INTERNET COMO FUENTE (CIBERPLAGIO)}

Los resultados obtenidos muestran cómo la práctica académica deshonesta de plagiar trabajos académicos (completos o partes) mediante Internet presenta una relación lineal inversa con la severidad de dicha práctica. Esto es, a mayor deshonestidad menor frecuencia con que se realiza el comportamiento, tanto declarado como atribuido al resto del alumnado.

\section{Gráfico 3}

Frecuencia declarada de plagio sobre Internet

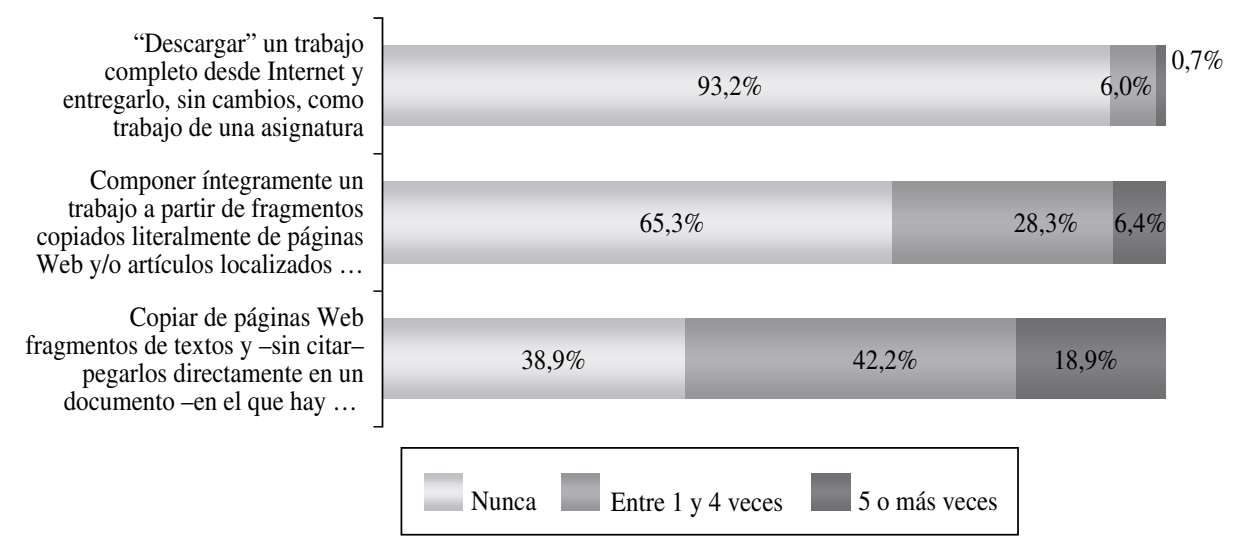

En particular, insertar fragmentos de texto copiados de páginas Web en trabajos propios sin citar aparece, tal y como recoge el gráfico 3, como la práctica de ciberplagio más recurrida por parte de los alumnos universitarios; más del $60 \%$ dice haberlo hecho alguna vez (42,2\% entre 1 y 4 veces, y el $18,9 \%$ más de 4 veces). Poco más del $85 \%$ del alumnado (24,2\% esporádicamente, y $62,4 \%$ frecuentemente o muy frecuentemente) cree que sus compañeros han realizado esta práctica (ver gráfico 4).

En el gráfico 4 aparece en segunda posición, en cuanto a actividad de ciberplagio cometida con mayor frecuencia, la completa composición de un trabajo académico a partir de fragmentos extraídos literalmente de Internet; un 34,7\% admite haber elaborado un trabajo académico de este modo (28,3\% entre 1 y 4 veces y el 6,4\% más de 4 veces). Este porcentaje se eleva hasta un $79 \%$ cuando los alumnos atribuyen dicha acción a sus compañeros; destacando aquí que casi la mitad del alumnado afirma que sus compañeros frecuentemente o muy frecuentemente elaboran los trabajos académicos de esta forma (ver gráfico 4).

La práctica llevada a cabo con menor frecuencia, dentro de este apartado, es la entrega de un trabajo completo "descargado" de la Red, y sobre el que no se ha realizado 
Gráfico 4

Frecuencia atribuida a los compañeros sobre la realización de plagio sobre Internet

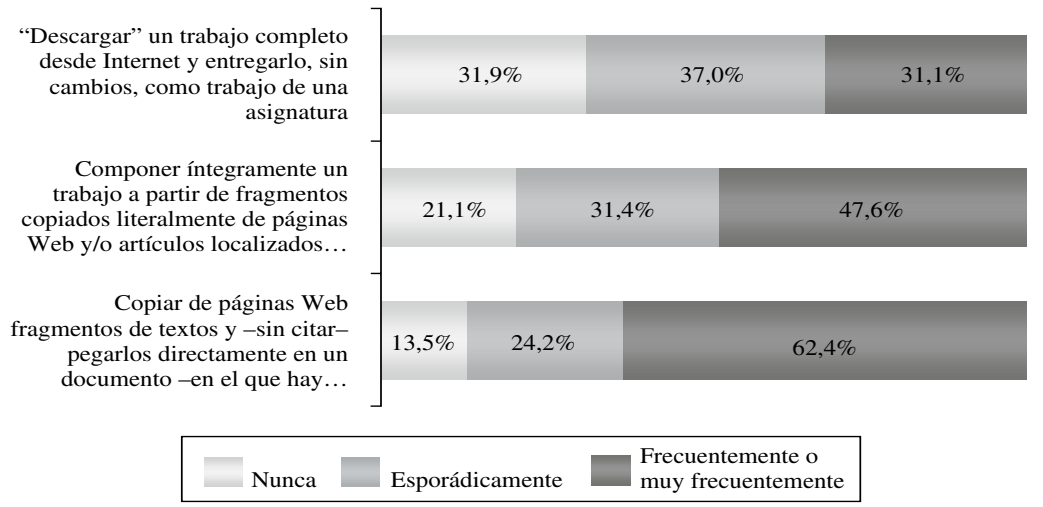

cambio alguno. Algo más del $6 \%$ dice haber entregado al menos un trabajo de esta manera, mientras que más del $68 \%$ del alumnado universitario atribuye a sus compañeros esta práctica (37\% esporádicamente y $31,1 \%$ frecuentemente o muy frecuentemente).

\section{PLAGIO DE FUENTES IMPRESAS}

Ante la cuestión de con qué frecuencia el alumnado suele copiar fragmentos de fuentes impresas tales como libros, periódicos, artículos de revista, etc., y usarlos sin citar para elaborar trabajos académicos propios, los resultados recogidos en los gráficos 5 y 6 muestran un resultado, cuando menos, curioso. Más de la mitad del alumnado dice que nunca ha plagiado de fuentes impresas, mientras que casi el $85 \%$ atribuye a sus compañeros la comisión de esta práctica, esporádicamente, al menos. Cabe destacar que más de la mitad atribuye este comportamiento como frecuente o muy frecuente.

\section{PLAGIO DE TRABAJOS ACADEMICOS}

En relación a los trabajos académicos, el plagio y/o autoplagio de partes de trabajos ya entregados, propios o ajenos, aparece como la práctica más frecuente; la mitad del alumnado declara haberla realizado alguna vez, y más del $80 \%$ cree que sus compañeros la realizan con frecuencia. Una distribución muy similar se observa en el acto de facilitar a otro alumno un trabajo de años anteriores para que lo entregue como inédito (ver gráficos 7 y 8 ).

Algo más de una cuarta parte del alumnado manifiesta haber entregado trabajos que ya habían sido librados con anterioridad, empatando técnicamente en el tercer lugar los de elaboración propia con los elaborados por otros compañeros (ver gráfico 7). Estas dos prácticas mantienen diferencias ínfimas entre sí en cuanto a la atribución de frecuencia con que las realizan los compañeros: en torno al $80 \%$ en ambos casos. 


\section{Gráfico 5}

Frecuencia declarada de plagio de fuentes impresas

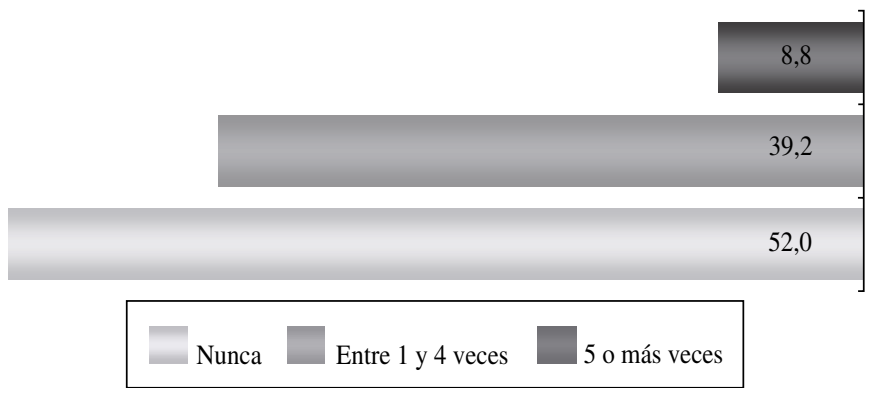

\section{Gráfico 6}

Frecuencia atribuida de plagio de fuentes impresas.

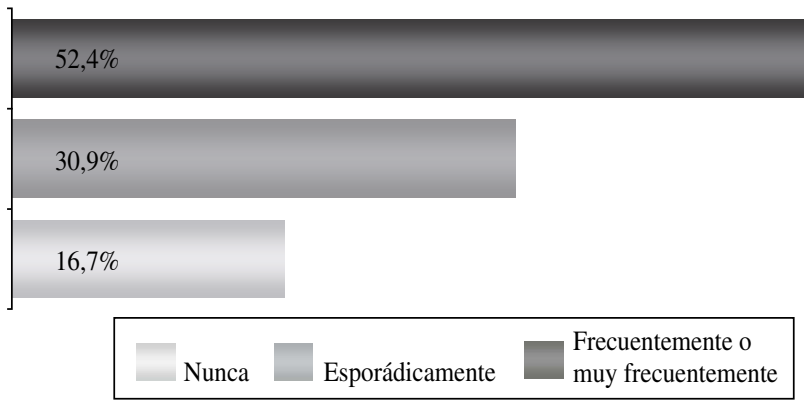

\section{Gráfico 7}

Frecuencia declarada de plagio de trabajos académicos

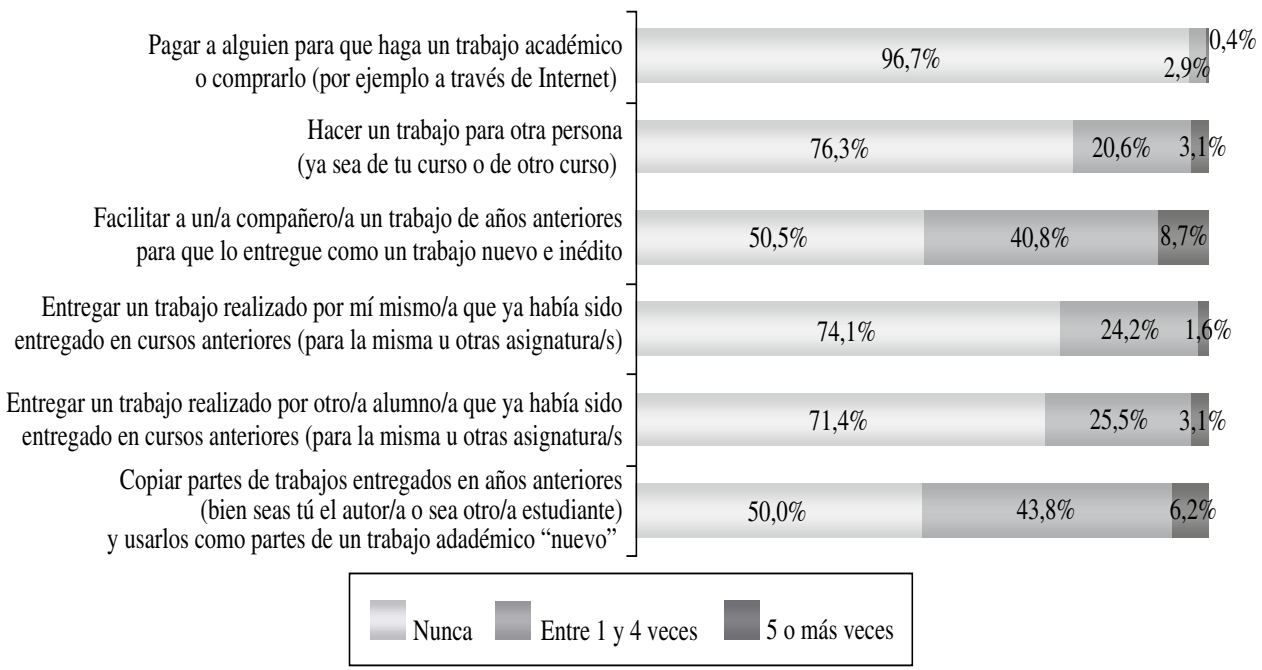


Las últimas posiciones, en cuanto a la comisión de fraude académico en la elaboración de un trabajo, vienen ocupadas por la realización expresa de trabajos con autoría de otra persona. En particular, alrededor de una cuarta parte de los estudiantes declara haber hecho trabajos académicos para otra persona (ver gráfico 7), y el $3 \%$ confiesa haber pagado a alguien para que le haga el trabajo académico, o sencillamente lo ha comprado. Estos resultados, como viene siendo habitual, difieren en gran medida de las atribuciones que el alumnado realiza para con sus compañeros (ver gráfico 8). Casi la mitad del alumnado cree que el resto de alumnado ha hecho alguna vez un trabajo para otra persona $(36,6 \%$ esporádicamente y $10,5 \%$ frecuentemente o muy frecuentemente), y algo más del $30 \%$ cree que sus compañeros han comprado trabajos o han pagado para que se los hagan (21,5\% esporádicamente y $8,7 \%$ frecuentemente o muy frecuentemente).

El hecho de que la compra de trabajos académicos ya realizados ocupe la última posición del ranking de deshonestidad académica en esta sección no debe hacernos perder de vista que quizás esta sea la modalidad fraudulenta que más crezca en el futuro inmediato. Es previsible que cuando las instituciones académicas españolas instauren, como han hecho las de otros países, sistemas de detección del plagio se produzca un gran incremento de la demanda de trabajos "a la carta", realizados según las necesidades del comprador y con garantías de superar la prueba de cualquier programa de detección (Comas \& Sureda, 2008).

\section{Gráfico 8}

Frecuencia atribuida a los compañeros de plagio de trabajos académicos

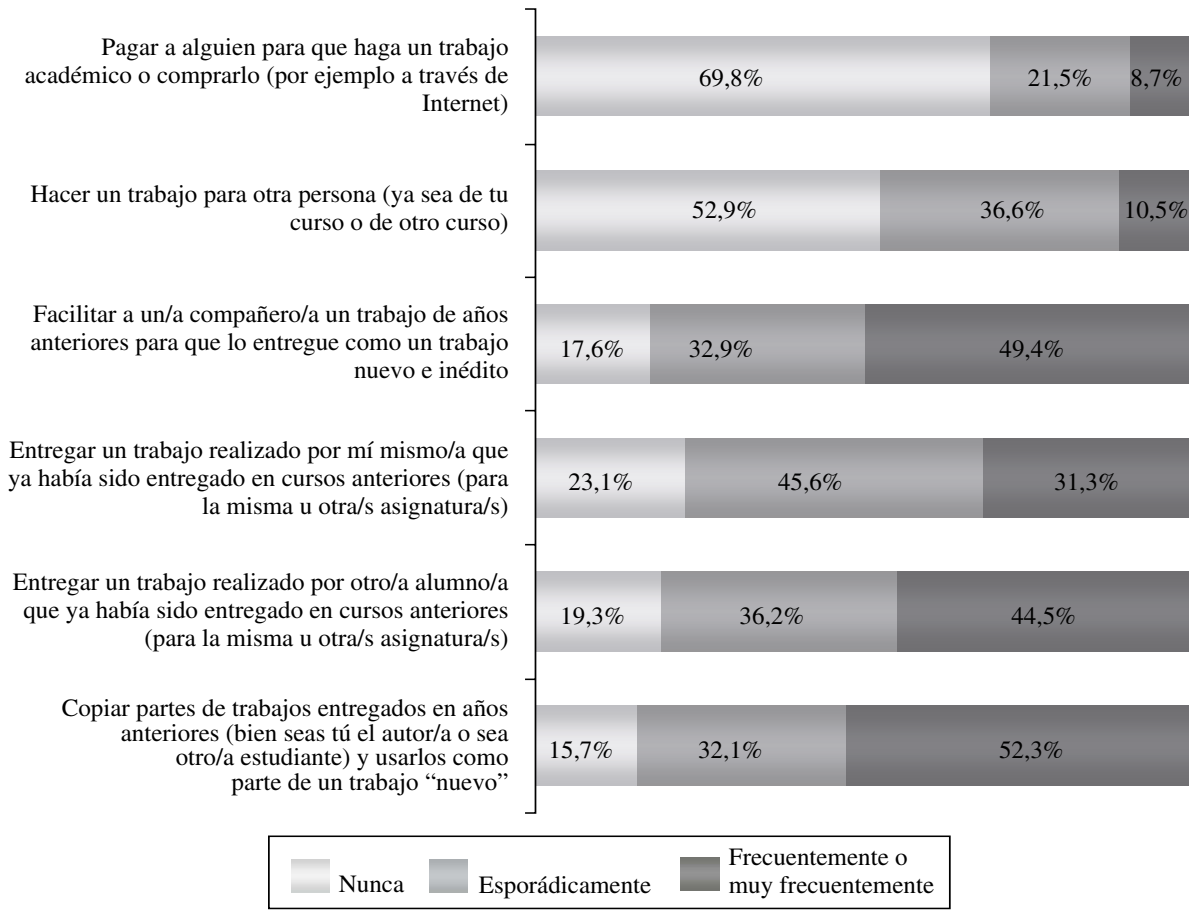


Más de la mitad de los estudiantes universitarios confiesan haber insertado en el apartado bibliográfico de un trabajo referencias que realmente no han consultado, tal y como recoge el gráfico 9 (40,3\% entre 1 y 4 veces, y 12,8\% 5 o más veces). Estos valores alcanzan niveles cercanos al $80 \%$ cuando se refieren a la opinión que tiene el alumnado preguntado acerca de si sus compañeros llevan a cabo este tipo de prácticas fraudulentas (ver gráfico 10).

Resulta curioso que en este caso, al igual que ocurriera en el caso del plagio de fuentes impresas, se invierte la relación entre la frecuencia declarada y la atribuida; casi la mitad del alumnado ha confesado que nunca ha referenciado bibliografía no consultada, cuando el $29,9 \%$ cree que sus compañeros han referenciado esporádicamente, y un considerable 49,7\% afirma que sus compañeros realizan este tipo de práctica frecuentemente o muy frecuentemente.

\section{Gráfico 9}

Frecuencia declarada de haber falseado la bibliografía

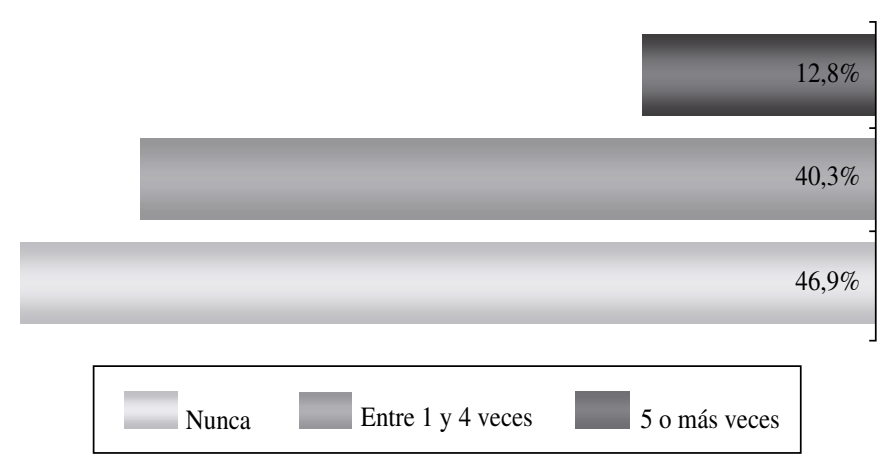

Gráfico 10

Frecuencia atribuida de haber falseado la bibliografía

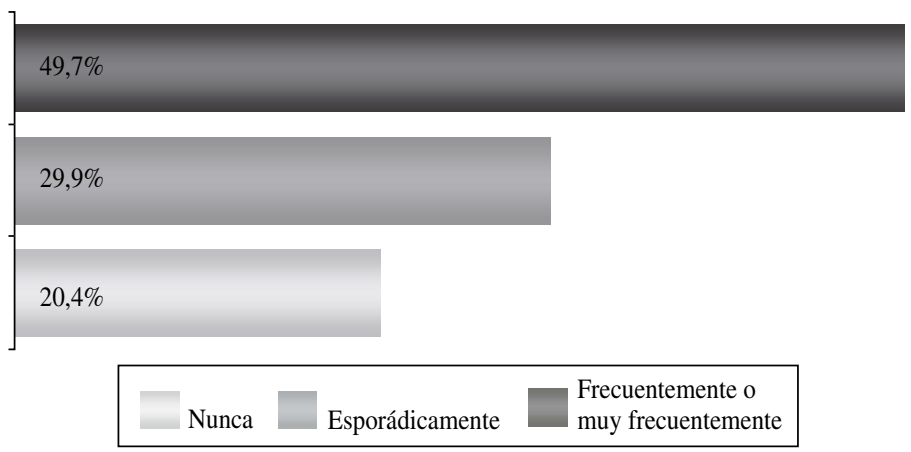


Centrando la atención en la fuente utilizada habitualmente para plagiar en la elaboración de trabajos académicos, algo más de tres cuartas partes del alumnado universitario $(77,2 \%)$ manifiesta que, desde su experiencia y conocimiento, se suelen utilizar con mayor frecuencia los recursos que encuentran en Internet para copiar trabajos o fragmentos de trabajos. El resto de fuentes empleadas a tal efecto son: los trabajos entregados con anterioridad, propios o ajenos $(15,5 \%)$, y los recursos impresos tales como los libros, las revistas y los periódicos $(7,3 \%)$.

\section{RANKING Y CONSISTENCIA DE LAS PRACTICAS ACADEMICAS DESHONESTAS}

La tabla 3 recoge, a modo de resumen, el porcentaje de alumnos que admite haber realizado (declarado) la práctica académica deshonesta citada en cada escenario (entre 1 y 4 veces y 5 o más veces) y el porcentaje de alumnado que considera (atribuido) que sus compañeros han realizado la práctica asociada al escenario (esporádicamente o frecuentemente o muy frecuentemente). Además, la tabla muestra la diferencia entre los dos porcentajes y los rangos de cada una de estas tres variables, ordenados de mayor a menor valor del porcentaje.

El porcentaje medio declarado es de 34,2\%; es decir, algo más de una tercera parte del alumnado, como media, dice llevar a cabo una o más de las prácticas aquí expuestas una o más veces. En cambio, la media del porcentaje atribuido es de 69,7\%; el doble de alumnos cree que sus compañeros realizan las citadas prácticas académicas deshonestas. La diferencia media entre el porcentaje declarado y el porcentaje atribuido es de 35,6\%. Aplicando a esta nueva variable una comparación de medias en un diseño de medidas repetidas, y por vía paramétrica, observamos significación estadística, por lo que la diferencia entre los porcentajes no se debe al azar. Es decir, la declaración de comisión de prácticas deshonestas académicas difiere significativamente de la creencia que sobre la comisión de dichas prácticas tienen los universitarios de sus compañeros $(\mathrm{t}=11.872$; g.l. $=16 ; \mathrm{p}<0.001)$.

Respecto a la consistencia del ranking de comisión de acciones contra la integridad académica, los resultados obtenidos a partir de la correlación entre el porcentaje declarado y el porcentaje atribuido informan de una muy alta relación lineal directa entre ambos porcentajes $(\mathrm{r}=0.839$; g.l. $=17$; $\mathrm{p}<0.001)$, indicando que, efectivamente, existe consistencia entre una ordenación y otra, permitiendo conceder credibilidad a que aquellas prácticas con un mayor número de alumnos que declaran su realización son también las prácticas más atribuidas a los compañeros.

De esta manera, dejar copiar a un compañero durante un examen ocupa la primera posición en cuanto a práctica deshonesta, tanto declarada como atribuida, que junto con la copia de fragmentos de páginas Web y de trabajos entregados anteriormente componen las tres actividades fraudulentas presentes entre las cinco primeras posiciones de ambas ordenaciones. Mayor consenso aún encontramos en las tres últimas posiciones, donde aparecen las tres mismas actividades, siendo la suplantación de otro alumno en un examen la práctica con un menor porcentaje, tanto declarado como atribuido; mientras 
que el uso de medios tecnológicos en el desarrollo de un examen, y pagar a alguien para la realización de un trabajo alternan la decimosexta y decimoquinta plaza entre las ordenaciones.

\section{Tabla 3}

Porcentajes y rangos de las prácticas académicas deshonestas

\begin{tabular}{|c|c|c|c|c|c|c|}
\hline Escenario & Declarado & $\mathrm{Rd}^{1}$ & Atribuido & $\mathrm{Ra}^{2}$ & Diferencia & $\operatorname{Rdif}^{3}$ \\
\hline $\begin{array}{l}\text { Dejar que otro estudiante me copie durante un } \\
\text { examen. }\end{array}$ & $74,40 \%$ & 1 & $88,82 \%$ & 1 & $14,43 \%$ & 17 \\
\hline $\begin{array}{l}\text { Copiar de páginas Web fragmentos de textos y sin } \\
\text { citar pegarlos directamente en un trabajo. }\end{array}$ & $61,10 \%$ & 2 & $86,55 \%$ & 4 & $25,45 \%$ & 14 \\
\hline $\begin{array}{l}\text { Insertar en el apartado bibliográfico de un trabajo } \\
\text { referencias que realmente no se han consultado. }\end{array}$ & $53,10 \%$ & 3 & $79,60 \%$ & 9 & $26,50 \%$ & 13 \\
\hline $\begin{array}{l}\text { Copiar partes de trabajos entregados en años anterio- } \\
\text { res y usarlos como partes de un trabajo académico } \\
\text { "nuevo". }\end{array}$ & $50,00 \%$ & 4 & $84,34 \%$ & 5 & $34,34 \%$ & 10 \\
\hline $\begin{array}{l}\text { Facilitar a un/a compañero/a un trabajo de años } \\
\text { anteriores para que lo entregue como un trabajo } \\
\text { nuevo e inédito. }\end{array}$ & $49,55 \%$ & 5 & $82,35 \%$ & 7 & $32,81 \%$ & 11 \\
\hline $\begin{array}{l}\text { Copiar fragmentos de fuentes impresas y usarlos sin } \\
\text { citar para elaborar trabajos académicos propios. }\end{array}$ & $47,99 \%$ & 6 & $83,30 \%$ & 6 & $35,31 \%$ & 8 \\
\hline Copiar de un/a compañero/a durante un examen. & $46,36 \%$ & 7 & $88,12 \%$ & 2 & $41,76 \%$ & 6 \\
\hline Utilizar "chuletas" en un examen. & $43,40 \%$ & 8 & $86,63 \%$ & 3 & $43,23 \%$ & 5 \\
\hline $\begin{array}{l}\text { Componer íntegramente un trabajo a partir de frag- } \\
\text { mentos copiados de páginas Web. }\end{array}$ & $34,74 \%$ & 9 & $78,95 \%$ & 10 & $44,20 \%$ & 4 \\
\hline $\begin{array}{l}\text { Entregar un trabajo realizado por otro/a alumno/a que } \\
\text { ya había sido entregado en cursos anteriores. }\end{array}$ & $28,57 \%$ & 10 & $80,73 \%$ & 8 & $52,16 \%$ & 2 \\
\hline Falsear datos en trabajos académicos. & $26,91 \%$ & 11 & $62,93 \%$ & 13 & $36,02 \%$ & 7 \\
\hline $\begin{array}{l}\text { Entregar un trabajo realizado por mi mismo/a que ya } \\
\text { había sido entregado en cursos anteriores. }\end{array}$ & $25,86 \%$ & 12 & $76,91 \%$ & 11 & $51,05 \%$ & 3 \\
\hline $\begin{array}{l}\text { Hacer un trabajo para otra persona (ya sea de tu } \\
\text { curso o de otro curso). }\end{array}$ & $23,67 \%$ & 13 & $47,09 \%$ & 14 & $23,42 \%$ & 15 \\
\hline $\begin{array}{l}\text { "Descargar" un trabajo completo desde Internet } \\
\text { y entregarlo, sin cambios, como trabajo de una } \\
\text { asignatura. }\end{array}$ & $6,78 \%$ & 14 & $68,10 \%$ & 12 & $61,33 \%$ & 1 \\
\hline $\begin{array}{l}\text { Pagar a alguien para que haga un trabajo académico } \\
\text { o comprarlo. }\end{array}$ & $3,27 \%$ & 15 & $30,22 \%$ & 16 & $26,95 \%$ & 12 \\
\hline $\begin{array}{l}\text { Utilizar nuevas tecnologías (móvil, auriculares } \\
\text { inalámbricos,...) para obtener las respuestas en un } \\
\text { examen. }\end{array}$ & $2,55 \%$ & 16 & $37,82 \%$ & 15 & $35,28 \%$ & 9 \\
\hline Hacer un examen en nombre de otra persona. & $2,38 \%$ & 17 & $22,82 \%$ & 17 & $20,44 \%$ & 16 \\
\hline
\end{tabular}




\section{LIMITACIONES, CONCLUSIONES Y DISCUSION}

El trabajo presentado adolece, por un lado, de las limitaciones propias del enfoque adoptado y, por otro, de cierta falta de completitud ${ }^{5}$ ya que, por limitaciones de espacio, no hemos podido incluir en este documento todos los datos obtenidos en nuestra investigación. A pesar de ello, el hecho de combinar en nuestro estudio acerca de la honestidad académica el método de análisis descrito como respuestas autorreferidas y el método de respuestas atribuidas mejora el rigor y la capacidad de certeza del análisis a desarrollar y responde a los objetivos plantedos.

Los datos obtenidos muestran que los universitarios españoles se mueven en frecuencias, tendencias y dinámicas muy parecidas a las observadas por otros estudios sobre el tema desarrollados en otros países (Baruchson-Arbib \& Yaari, 2004; Bilic-Zulle, 2004; Chapman \& Lupton, 2004; Carpenter, Harding, Finelli, Montgomery, \& Passow, 2006; Ellery, 2008; Lambert et al., 2003; Lin \& Wen, 2007; McCabe, 2001; Seppänen, 2002; Teixeira \& Rocha, 2006; Underwood \& Szabo, 2003) y también a los realizados en universidades españolas (Agnes, 2008; Rey-Abella et al., 2006; Sureda et al., 2008).

A la hora de realizar trabajos académicos, Internet se ha convertido en la fuente principal (casi única) de documentación académica para el alumnado universitario (Sureda \& Comas, 2005). Parece entonces lógico que también sean Internet y los recursos asociados a ésta las principales fuentes de plagio académico. El libro se ha visto reemplazado por la Red como fuente documental y, por lo que parece, también como fuente de plagio. La facilidad y comodidad de acceso a las posibilidades que confieren las TIC, el anonimato que confiere el medio, la novedosa concepción de autoría y propiedad intelectual que parece haber nacido al amparo de dichas tecnologías y las posibilidades que se presentan de copiar y pegar fácilmente gran cantidad de documentos con los procesadores de texto actuales hacen que el ciberplagio presente mayor prevalencia que otros tipos de plagio entre los universitarios de nuestro país -al igual que sucede en la mayoría de países de características de desarrollo parecidas-.

Los resultados obtenidos acerca de las prácticas deshonestas llevadas a cabo en el desarrollo de pruebas escritas muestran que en este aspecto las TIC todavía no han modificado en gran medida las prácticas "tradicionales" tal y como sí sucede en el caso de la elaboración de trabajos. Las chuletas y el vistazo al examen del compañero continúan siendo las estrategias estrella a la hora de copiar en exámenes. Aun así, advertimos del potencial de los nuevos medios tecnológicos para cometer fraudes en el transcurso de exámenes y sugerimos que se preste atención a la evolución de su uso en los siguientes años.

En lo que respecta a las perspectivas de futuro del tema, queremos hacer mención a dos aspectos que nos parecen básicos: a) la necesidad de nuevas investigaciones basadas en la combinación de aproximaciones cuantitativas y cualitativas al fenómeno, así como el análisis de las causas asociadas a éste; y b) la conveniencia de que las autoridades académicas asuman el reto de plantear soluciones a la situación aquí descrita. Las universidades españolas deben asumir el reto que plantean algunos de los datos aportados.

5 Nos referimos a cuestiones tales como: causas asociadas a la comisión de plagio académico, valoración moral por parte del alumnado de las prácticas académicamente deshonestas, idioma/s en que se plagian trabajos, nivel del sistema educativo en que se dan más casos de plagio académico, etc. 
Los valores de la honestidad confianza, responsabilidad, respeto y equidad no pueden quedar al margen de los objetivos formativos de los centros de enseñanza superior.

A modo de epílogo de nuestra propuesta, nos parece interesante el plantear abiertamente una serie de cuestiones que pueden guiar futuras aproximaciones al tema abordado y dotarle de mayor profundidad:

- ¿Tenemos una universidad casi-decimonónica en su planteamiento, finalidad, funcionamiento e ideología enclavada en una realidad altamente "tecnologizada" propia del XXI y de la Sociedad de la Información y Conocimiento? ¿Se ha avanzado suficientemente en introducir nuevas estrategias y metodologías de enseñanza-aprendizaje en el sistema educativo adaptadas a la nueva realidad nacida al amparo de la Era Digital? ¿Qué cambiará al respecto del tema abordado (si algo cambia) la implantación del Plan Bolonia a partir del próximo curso académico?

- ¿Qué porción de culpa puede tener el fenómeno de la masificación en la universidad en el avance de las prácticas académicamente deshonestas?

- ¿Dan, de manera general, buen ejemplo los docentes a su alumnado en cuanto a los principios que deben marcar la integridad académica?

- ¿Por qué si los datos son tan sangrantes como los que demuestra este trabajo y otros similares, no se implementan medidas en nuestro país para mejorar la situación ${ }^{6}$ ? ¿Es posible que todo lo relatado en estas páginas no se intente remediar por intentar mantener una especie de pacto de silencio entre alumnado y profesorado que ayude al mantenimiento de cierto statu quo en la institución universitaria?

- ¿Cómo influye, si lo hace en alguna medida, la comisión de prácticas deshonestas en la universidad con el desarrollo futuro de la labor profesional para la que el alumno se ha formado?

- La universidad española: ¿tiende al modelo del "fast food learning”? o empleando el concepto de Modernidad líquida de Bauman (2003): ¿tiende a convertirse en una "universidad líquida"?

\section{REFERENCIAS BIBLIOGRAFICAS}

Agnes, J. (2008). Los usos de Internet en la educación superior: de la documentación al plagio. Six Degrés. Recuperado el 12 de junio de 2008 de http://www.compilatio.net/files/080521_ sixdegres-univ-barcelona_univ-zaragoza_encuesta-plagio.pdf

Ashworth, P., Bannister, P. \& Thorne, P. (1997). Guilty in whose eyes? University students' perceptions of cheating and plagiarism in academic work and assessment. Studies in Higher Education, 22 (2), 187-203.

Baruchson-Arbib, S. \& Yaari, E. (2004). Printed Versus Internet plagiarism. International Journal of Information Ethics, 1 (6).

Bauman, Z. (2003). Modernidad líquida. Fondo de Cultura Económica, México DF.

Bilic-Zulle, L. (2004). Prevalence and attitudes toward plagiarism among medical students. Tesis Doctoral. Presentación accesible desde: http://mi.medri.hr/assets/LBZ\%20Disertation.pps $(02 / 05 / 08)$

6 Existe una iniciativa pionera nacida en la Universidad de Granada a raíz de la creación de la llamada "Plataforma anti-plagio". 
Bowers, W.J. (1964). Student dishonesty and its control in college. New York: Bureau of Applied Social Research.

Brimble, M. \& Stevenson-Clarke, P. (2005). Perceptions of the prevalence and seriousness of academic dishonesty in Australian universities. Australian Educational Researcher, 32 (3), 19-44.

Carpenter, D., Harding, T.S., Finelli, C.J., Montgomery, S.M. \& Passow, H.J. (2006). Engineering students' perceptions of and attitudes towards cheating. Journal of Engineering Education, 95, 181-194.

Chapman, K.J. \& Lupton, R.A. (2004). Academic Dishonesty in a Global Educational Market: A Comparison of Hong Kong and American university business Students. The Internacional Journal of Educational Management, 18 (7). Vol. 425-435.

Comas, R. (2009). El ciberplagio y otras formas de deshonestidad académica entre los universitarios. Tesis Doctoral no publicada, Universidad de las Islas Baleares, Palma de Mallorca, España.

Da-Costa, I. et al. (2006). Má Prática Acadêmica por Estudantes de Medicina: Estudo Piloto. Gazeta Médica da Bahia, 76 (2), 29-37.

Davis, F., Pierce, M., Yandell, L. \& Arnow, P. (1995). Cheating in college and the type A personality: a reevaluation. College Student Journal, 29 (4).

Diekhoff, G.M., LaBeff, E.E., Clark, R.E., Williams, L.E., Francis, B. \& Haynes, V.J. (1996). College cheating: Ten years later. Research in Higher Education, 37, 487-502.

Ellery, K. (2008). Undergraduate plagiarism: a pedagogical perspective. Assessment \& Evaluation in Higher Education, 8.

García-Barbastefano, R. \& Gomes-de-Souza, C. (2007). Percepção do conceito de plágio acadêmico entre alunos de engenharia de produção e ações para sua redução. Revista Produção, edición especial diciembre. Artículo extraído de los Anais do XXVII Encontro Nacional de Engenharia de Produção. Universidade Federal de Santa Catarina. Recuperado el 07 de mayo de 2010 de: http:// producaoonline.org.br/index.php/rpo/article/view/52/52

Genereux, R.L. \& McLeod, B.A. (1995). Circumstances surrounding cheating: A questionnaire study of college students. Research in Higher Education, 36, 687-704.

Haines, V.J., Diekhoff, G.M., LaBeff, E.E. \& Clark, R.E. (1986). College cheating: immaturity, lack of commitment, and the neutralizing attitude. Research in Higher Education, 25.

Harding, T.S., Carpenter, D.D., Montgomery, S.M., \& Stenech, N.H. (2001). The Current State of Research on Academic Dishonesty Among Engineering Students. Ponencia presentada en la 31st ASEE/IEEE Frontiers in Education Conference, Reno, NV.

Harding, T.S., Mayhew, M.J., Finelli, C.J. \& Carpenter, D.D. (2007). The theory of planned behavior as a model of academic dishonesty in humanities and engineering undergraduates. Journal on Research and Teaching, 17.

Hinman, M. (2002). Academic integrity and the world wide web. Computers and Society, Vol. 31 (1).

Jeffers, B.R. (2002). Continuing education in research ethics for the clinical nurse. The Journal of Continuing Education in Nursing, 33 (6), 265-269.

Joyce, D. (2007). Academic Integrity and Plagiarism: Australasian perspectives. Computer Science Education, 17 (3).

Kibler, W.L. (1993). Academic dishonesty: A student development dilemma. NASPA Journal, 30, 252-267.

Lambert, E., Hogan, N. \& Barton, S. (2003). Collegiate academic dishonesty revisited: what have They done, how often have They done it, who does it, and why did They do it? Electronic Journal of Sociology, 6.

Lin, T. \& Wen, N. (2007). Academic dishonesty in higher education: A nationwide Study in Taiwan. Higher Education: The International Journal of Higher Education and Educational Planning, 54 (1), 85-97. 
McCabe, D.L. (2001). Cheating in academic institutions: a decade of research. Ethics and Behavior, 11.

McCabe, D.L. \& Trevino, L.K. (1993). Academic dishonesty: Honor codes and other contextual influences. Journal of Higher Education, 64, 520-538.

Mejía, J.F. y Ordóñez, C.L. (2004). El fraude académico en la Universidad de los Andes: ¿Qué tanto y por qué?. Revista de Estudios Sociales, 18, 13-25.

Moeck, P.G. (2002). Academic dishonesty: Cheating among community college students. Community College Journal, 26, 479-491.

Rey-Abella, F., Blanch, C. y Folch-Soler, A. (2006). Nivel de conducta académica deshonesta entre los estudiantes de una escuela de ciencias de la salud. Enfermería Clínica, 16 (2), 57-61.

Rogers, C. (2006). Faculty perceptions about e-cheating during online testing. Journal of Computing Sciences in Colleges, 12 (3).

Saldaña, C.; Quezada, J.J.; Peña, A. \& Mayta, P. (2010). Alta frecuencia de plagio en tesis de medicina de una universidad pública peruana. Rev Peru Med Exp Salud Publica, 27 (1), 63-67.

Seppänen, M. (2002). Finns target master plagiarists. Times Higher Education Supplement, 1 February, p. 11. Recuperado el 5 de junio de 2008 de http://www.timeshighereducation. co.uk/story.asp?storyCode $=166956 \&$ sectioncode $=26$

Sureda, J. \& Comas, R. (2005). Fuentes de información y documentación a través de Internet para investigadores en educación ambiental. Madrid: Ministerio de Medio Ambiente. Serie de Educación Ambiental.

Sureda, J. et al. (2008). El plagio y otras formas de deshonestidad académica entre el alumnado de la Universitat de les Illes Balears: resultados generales, por género y por ramas de estudios. Recuperado el 16 de junio de 2008 de http://ciberplagio.es/attachment.php?key = 38

Teixeira, A.C. \& Rocha, M.F. (2006). Academic Cheating in Austria, Portugal, Romania and Spain: a comparative análisis. Research in Comparative and International Education, 1 (3).

Tibbetts, S.G. (1998). Differences between criminal justice majors and noncriminal justice majors in determinants of test cheating intentions. Journal of Criminal Justice Education, 9, 81-94.

Underwood, J. \& Szabo, T. (2003). Academic offences and e-learning: individual propensities in cheating. British Journal of Educational Technology, 34, 467-478.

University of San Diego (1997). Fundamental principles of academic integrity, San Diego University Ethics Updates. Recuperado el 16 de junio de 2008 de http://ethics.sandiego.edu/ eac/Summer2000/Readings/Principles.html

Whitley, B.E. (1998). Factors associated with cheating among college students: A review. Research in Higher Education, 39, 235-274. 
